\title{
Oxidative Stress and Epigenetic Regulation in Ageing and Age-Related Diseases
}

\author{
Chiara Cencioni ${ }^{1}$, Francesco Spallotta ${ }^{1}$, Fabio Martelli ${ }^{2}$, Sergio Valente $^{3}$, Antonello Mai ${ }^{3}$, \\ Andreas M. Zeiher ${ }^{4}$ and Carlo Gaetano ${ }^{1, *}$
}

1 Division of Cardiovascular Epigenetics, Department of Cardiology, Goethe University, Frankfurt am Main 60596, Germany; E-Mails: pitini@hotmail.it (C.C.); fspallotta@gmail.com (F.S.)

2 Molecular Cardiology Laboratory, IRCCS-Policlinico San Donato, San Donato Milanese, Milan 20097, Italy; E-Mail: fabio.martelli@grupposandonato.it

3 Pasteur Institute-Cenci-Bolognetti Foundation, Department of Drug Chemistry and Technologies, Sapienza University of Rome, Rome 00185, Italy; E-Mails: sergio.valente@uniroma1.it (S.V.); antonello.mai@uniroma1.it (A.M.)

4 Internal Medicine Clinic III, Department of Cardiology, Goethe University, Frankfurt am Main 60596, Germany; E-Mail: zeiher@em.uni-frankfurt.de

* Author to whom correspondence should be addressed; E-Mail: gaetano@em.uni-frankfurt.de; Tel.: +49-69-6301-87963; Fax: +49-69-6301-4037.

Received: 21 May 2013; in revised form: 19 August 2013 / Accepted: 21 August 2013 /

Published: 28 August 2013

\begin{abstract}
Recent statistics indicate that the human population is ageing rapidly. Healthy, but also diseased, elderly people are increasing. This trend is particularly evident in Western countries, where healthier living conditions and better cures are available. To understand the process leading to age-associated alterations is, therefore, of the highest relevance for the development of new treatments for age-associated diseases, such as cancer, diabetes, Alzheimer and cardiovascular accidents. Mechanistically, it is well accepted that the accumulation of intracellular damage determined by reactive oxygen species (ROS) might orchestrate the progressive loss of control over biological homeostasis and the functional impairment typical of aged tissues. Here, we review how epigenetics takes part in the control of stress stimuli and the mechanisms of ageing physiology and physiopathology. Alteration of epigenetic enzyme activity, histone modifications and DNA-methylation is, in fact, typically associated with the ageing process. Specifically, ageing presents peculiar epigenetic markers that, taken altogether, form the still ill-defined "ageing epigenome". The comprehension of mechanisms and pathways
\end{abstract}


leading to epigenetic modifications associated with ageing may help the development of anti-ageing therapies.

Keywords: epigenetics; ageing; oxidative stress; cardiovascular; endothelial; cardiac

\section{Introduction}

Ageing is a multidimensional irreversible accumulation of physical, environmental and social changes. Nowadays, ageing biology and pathobiology are emerging as one of the most compelling areas of biomedical research, owing to current demographic trends and associated healthcare costs in Western societies [1,2]. Both the exponential growth of the literature on ageing during the last few years and the new "-omic" technology development for the study of lifespan revealed a great deal of interest in ageing and ageing-associated diseases among a large number of academic scientists and industrial entities [1]. From the onset of reproductive maturity, throughout the organism's life, the efficiency of various physiological processes progressively declines [3,4]. The gradual loss of homeostatic mechanisms associated with ageing is hypothetically due to an accumulation of molecular oxidative damage [5-7]. Indeed, the "Free Radical Theory of Ageing" is based on oxygen toxicity $[5,8]$. Molecular oxygen is a bi-radical able to generate partially reduced molecules and, then, reactive oxygen species (ROS). ROS can be detoxified within the cell by several kinds of antioxidants, based on both enzymatic and non-enzymatic mechanisms [5,8-10]. Examples of intracellular antioxidant enzymes are: superoxide dismutase (SOD), catalase, glutathione peroxidase, peroxiredoxin and sulfiredoxin $[5,8,10,11]$, whereas examples of low molecular weight antioxidants are: glutathione, vitamin C, vitamin A and vitamin E [5,8,10,11]. When all these endogenous antioxidants are insufficient, ROS increase, altering the cell normal redox state and, thus, provoking oxidative stress. High ROS levels cause toxic effects in the cell, because they are potentially detrimental for biological macromolecules, such as lipids, nucleic acids and proteins (Figure 1) [5,9]. In mammalian cells, ROS are mainly produced during physiological processes, such as cellular respiration, the activation of the arachidonic acid cascade and by several enzymes, including, for example, cytochrome p450, Nicotinamide Adenine Dinucleotide (NADH)/Nicotinamide Adenine Dinucleotide Phosphate (NADPH) oxidase and nitric oxide synthase $[5,9]$.

As a consequence of ROS accumulation, oxidative stress, the imbalance of the normal redox state, increases exponentially with age, paralleled by a remarkable decline of the cell repair machinery [10] (Figure 1). Oxidative stress contributes to the pathogenesis of several cardiovascular, pulmonary and neuronal disorders common among elderly people, such as myocardial infarction, diabetes, atherosclerosis, chronic obstructive pulmonary disease (COPD) or Alzheimer's disease [11-13].

Very recent studies indicated the multifactorial etiology of ageing-associated diseases as related to both genetic and epigenetic changes in the genome [14]. Although at the very beginning, scientists focused primarily on the genetic component of ageing, we would like to stress here that epigenetic mechanisms involved during ageing may play important physiopathological roles above all in the presence of oxidative stress (Figure 1). 
Figure 1. Oxidative stress, epigenetics and ageing. ROS, reactive oxygen species.

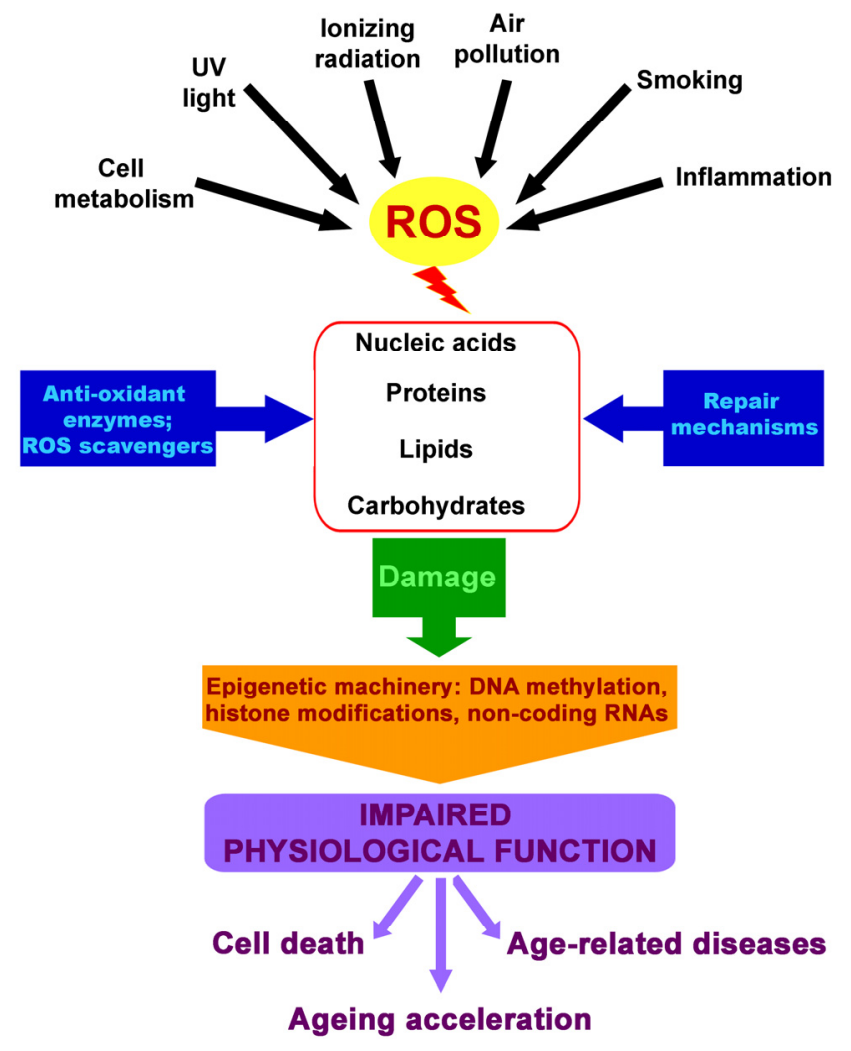

\section{The Epigenetic Machinery}

Epigenetics studies the somatically-acquired and, in some cases, trans-generationally inherited modifications of chromatin able to alter gene expression without changing the DNA blue-print $[15,16]$. Epigenetic mechanisms may act both qualitatively, to induce flexible, short-term gene silencing (histone tail modifications), and quantitatively, to provoke more stable, long-term gene expression (DNA methylation) [15]. The so-called epigenome control, in fact, relies on a large number of histone-modifying complexes, DNA methylation enzymes and non-coding RNAs, which, to a different extent, regulate chromatin structure [17].

Histones can be modified at many sites where the principal covalent modifications are: acetylation, phosphorylation, methylation, isomerization, ubiquitination and sumoylation (see, for review, [17]). Modified histone residues constitute the docking site for distinct chromatin-binding proteins, which direct the dynamic transition between transcriptionally active (euchromatin) and transcriptionally silent (heterochromatin) chromatin as a consequence of the covalent modification to which they are bound. The reversible nature of histone modifications accounts for the presence of chromatin remodeling enzymes with opposite functions, which allow chromatin to have a dynamic structure: for example, the histone acetyltransferases (HATs) and their counterpart, the histone deacetylases (HDACs), or the histone methyltransferases (HMTases), and their opposite, the histone demethylases [17].

DNA methylation is a very important epigenetic modification of cytosine residues in the primary DNA sequence. It is used by the cell as an epigenetic signal to lock genes in the so-called "off" position. Methylation plays an important role during numerous processes, including embryonic development, genomic imprinting, X-chromosome inactivation and the preservation of chromosome 
stability [18,19]. Specifically, during DNA methylation, a methyl group is added to the carbon-5 position of the cytosine pyrimidine ring by DNA methyltransferases to form 5-methylcytosine (5-MeC) [18,19]. DNA methylation occurring at promoter regions typically represses gene transcription by maintaining chromatin in a closed state $[18,19]$. This is achieved by recruiting methyl-CpG-binding-domain protein complexes that also contain HDACs. These complexes remove acetyl groups from the histone's $\mathrm{N}$-terminal ends and keep the chromatin in a closed configuration inaccessible to transcription factors and co-activators $[18,20]$. In contrast, the absence of 5-MeC at un-methylated promoters permits acetylation of histones (via HATs), which, in turn, allows a number of transcription activator complexes [20] to directly access chromatin and to promote transcription of a specific genomic region.

Non-coding RNAs, such as microRNAs, small interfering RNAs and long-non-coding RNAs, represent an additional layer of epigenetic control of gene expression [21]. They play a pivotal role in the regulation of gene transcription, through the recruitment of chromatin modification complexes, including the polycomb group complex [21].

\section{Epigenetic Traits of Ageing}

The entire epigenetic machinery, hitting specific targets and markers, might orchestrate cellular and organismal homeostasis. Alteration of epigenetic mechanisms may lead to accumulation of functional errors and to ageing-associated diseases, such as cancer. Indeed, aged organisms present a peculiarly modified epigenome (Table 1).

\subsection{Chromatin Alterations}

A large body of literature shows that the global hypomethylation occurring in an aged genome is often associated with a decrease in the activity of DNA methylation enzymes [22] with some peak of hyper methylation in specific gene loci, such as c-fos [23], IGF-II [24] and pl6ink4a [25]. Furthermore, ageing is characterized by specific histone modifications (Table 1). Histone acetylation on lysine 16 of histone H4 (H4K16) increases gradually, due to a reduction of sirtuin 1 (SIRT1) deacetylase protein level [26-28]. The histone methylation pattern is also sensitive to age: methylation of histones $\mathrm{H} 3$ and H4 changes, and depending on residues, it may decrease or increase [29]. The most relevant modified residues affected by the ageing-dependent decrease of the methylation state are: the tri-methylated lysine 36 of histone $\mathrm{H} 3$ (H3K36me3), the tri-methylated lysine 9 of histone H3 (H3K9me3) and the mono-methylated lysine 20 of histone H4 (H4K20me) [30]. Among residues affected by an increase of methylation, there are: the tri-methylated lysine 27 of histone H3 (H3K27me3) [30], the mono-/di-methylated lysine 79 of histone H3 (H3K79me/me2) [30] and the tri-methylated lysine 20 of the histone H4 (H4K20me3) [31,32]. Histone modification alterations are linked to changes in the expression level of epigenetic enzymes. Specifically, the, i.e., two histone methylation complexes, the polycomb repressive complex member EZH2 (PRC2) and the polycomb repressive complex member Bmi1 (PRC1) [26], decrease with age, whereas the histone demethylase jumonji domain containing 3 (JMJD3) increases (see Table 1) [33,34].

Senescence-associated heterochromatin foci (SAHFs) are one of cellular senescence markers more easily detectable in mice at the chromatin level. SAHFs are DNA domains that may be recognized 
when densely stained by 4',6-diamidino-2-phenylindole (DAPI) (Table 1) [35,36]. These peculiar heterochromatin structures increase in stress-induced senescent cells, where the activation of the cell cycle control $\mathrm{Rb}-\mathrm{p} 16^{\mathrm{ink} 4 \mathrm{a}}$ pathway contributes to inducing cell growth arrest. In addition, SAHFs associate with regions of transcriptional repression in which $\mathrm{H} 3 \mathrm{~K} 9 \mathrm{me} 3$ accumulates [35,37]. The presence of SAHFs seem to play a causal role in cellular senescence, because they induce repression of the E2F transcription factor family, fundamental for the progression of the cell cycle, and cause interruption of cell cycle progression [35].

Table 1. Epigenetic traits of ageing.

\begin{tabular}{ccc}
\hline Epigenetic ageing marker & Regulation & Reference \\
\hline Global DNA methylation & Decreased & {$[22]$} \\
DNA methylase activity & Decreased & {$[22]$} \\
PRC1, PRC2 & Decreased & {$[26]$} \\
SIRT1 & Decreased & {$[22,26,27]$} \\
miR-71 & Decreased & {$[30]$} \\
H3K36me3, H3K9me3, H4K20me & Decreased & {$[38]$} \\
c-fos, IGF-II, p16Ink4a methylation & Increased & {$[23-25]$} \\
H4K16ac & Increased & {$[22,26,27]$} \\
JMJD3 & Increased & {$[33,34]$} \\
H3K27m20me3 3 HK79me/me2 & Increased & {$[30]$} \\
SAHFs & Increased & {$[31,32]$} \\
mir-29 & Increased & {$[35-37]$} \\
mir-34a & Increased & {$[39-41]$} \\
mir-200 family & Increased & {$[40-43]$} \\
\hline
\end{tabular}

Notes: PRC1, polycomb-group repressive complex 1; PRC2, polycomb-group repressive complex 2; SIRT1, sirtuin 1; H3K36me3, tri-methylated lysine 36 of histone H3; H3K9me3, tri-methylated lysine 9 of histone H3; H4K20me, mono-methylated lysine 20 of histone H4; miR-71, micro-RNA 71; c-fos, FBJ murine osteosarcoma viral oncogene homolog; IGF-II, insulin-like growth factor II; p16Ink4a, cyclin-dependent kinase inhibitor 2A; H4K16ac, acetylated lysine 16 histone H4; JMJD3, histone demethylase jumonji domain containing 3; H3K27me3, tri-methylated lysine 27 of histone H3; H3K79me/me2, mono-/di-methylated lysine 79 of histone H3; H4K20me3, tri-methylated lysine 20 of the histone H4; SAHFs, senescence-associated heterochromatin foci; miR-29, micro-RNA 29; miR34a, micro-RNA 34a; miR-200, micro-RNA 200 family.

\section{2. miRNA Role in Ageing}

Several miRNA clusters are up- or down-modulated in different tissues during ageing and are able to hit molecular targets that regulate lifespan, such as insulin-like growth factor 1 (IGF1)/insulin [45], forkhead box, sub-group O (FOXO) [46], SIRT1 [47] and cyclin-dependent kinase inhibitor 1A (p21) (Table 1) [48]. Among miRNAs that affect longevity, in C. elegans, micro-RNA 71 (miR-71) acts to increase resistance to heat shock and oxidative stress [38]. Alteration in micro-RNA expression may be involved in the age-associated impairment of organ function often seen in elderly people. The vascular impairment observed during ageing, in fact, is often combined with the altered expression of several micro-RNAs, such as miR-29 [39-41], miR-34a [40-42], miR-217 [40,41] and miR-146 [41,49,50]. miR-29 is upregulated by transcriptional and post-transcriptional mechanisms seen in cultured 
senescent endothelial cells $[40,41]$ and in old mouse aortas, determining the reduction of extracellular matrix deposition and aneurysm formation [39]. miR-34a has been found upregulated both in vitro and in vivo, associated with the inhibition of cell proliferation, with a subsequent induction of cellular senescence and premature death, both in endothelial progenitor and mature cells $[40,41,43]$. In our studies about the effect of oxidative stress on human umbilical vein endothelial cells (HUVECs), we found that ROS induce expression of miR-200 family members [44]. Specifically, we observed that the increase in miR-200c expression upon oxidative stress determined the down-modulation of the zinc finger E-box binding homeobox 1 (Zeb1) transcription factor paralleled by apoptosis and senescence [44].

Cardiac ageing is characterized by cardiomyocyte cell death, hypertrophy and fibrosis, which is also regulated by miRNA alteration. Recently, Boon and coworkers demonstrated the contributive role of miR-34a in the age-dependent decline of cardiac function [42]. Specifically, they found that miR-34a is upregulated in the heart during ageing, determining, via repression of its target, PNUTS, telomere erosion, DNA damage and cardiomyocytes apoptosis [42]. The authors further demonstrated that the miR-34a-PNUTS axis rules ischemia reperfusion injury after acute myocardial infarction, a phenomenon strictly associated with oxidative stress damage [42].

\section{ROS, Epigenetics and Diseases}

Cardiovascular diseases are by far the leading cause of morbidity and mortality in industrialized nations [51]. Due to remarkable progress in prevention and acute cardiac patient care, cardiovascular diseases nowadays manifest significantly later in life [51]. Therefore, the incidence of coronary artery disease, myocardial infarction and heart failure, often strictly interconnected, increases almost exponentially with age [51]. Ageing affects cardiovascular tissues, introducing typical markers: aged hearts show hypertrophy and fibrosis, whereas the aged vasculature is affected by arterial thickening and increased stiffness [52]. In this light, the health of cardiac and arterial systems is not mutually exclusive, as each system greatly affects the other [52]. For instance, an increase in arterial stiffness leads to compensatory mechanisms by the myocardium, which includes left ventricular hypertrophy and fibroblast proliferation [53]. Therefore, physiological modifications may determine age-related physiopathological changes, such as vascular dysfunction or insufficient vascular growth and remodeling (hypertension). Heart fibrosis and hypertrophy induce slow propagation of electric impulse throughout the heart, modifying heart rate and the electrical impulse conduction, which increases the incidence of arrhythmias [54]. At the molecular level, ageing is associated with changes in the activity of a series of enzymes necessary for cardiovascular homeostasis. For example, aged endothelial cells exhibit a decrease in endothelial nitric oxide synthase (eNOS) activity and nitric oxide (NO) production [53]. NO is a gaseous molecule able to regulate vasodilatation, shear stress and vascular tone and to prevent thrombotic events and vascular inflammation [55].

The production of ROS increases during ageing and determines oxidative stress, which might be responsible for the SIRT1, a class III histone deacetylases, decreased activity and protein levels [56]. SIRT1 antagonization is involved in senescence of mouse fibroblasts, human cancer cells and endothelial cells [57]. Specifically, Ota and co-workers [57] found that SIRT1 chemical inhibition by sirtinol, or genetically by siRNA gene knockdown, induces a senescence-like phenotype in HUVECs. Specifically, SIRT1 inhibition determines an increase of p53 acetylation with a consequent growth 
arrest of endothelial cells. On the other hand, SIRT1 overexpression in HUVECs prevented premature senescence in the presence of high levels of hydrogen peroxide $\left(\mathrm{H}_{2} \mathrm{O}_{2}\right)$. Therefore, SIRT1 results play a pivotal role in the modulation of stress stimuli, at least, in part, via p53 deacetylation [58].

Endothelial cell senescence is associated with endothelial dysfunction and vulnerability to atherosclerotic lesions. As mentioned above, NO is fundamental for endothelial function. In line with this observation, Ota et al. [59] demonstrated that treatment with cilostazol, a phosphodiesterase 3 (PDE3) inhibitor, induced NO production, thanks to an increased level of cyclic adenosine monophosphate (cAMP) and a consequent eNOS phosphorylation by cAMP/cAMP dependent protein kinase (PKA) and phosphatidylinositol-4,5-bisphosphate 3-kinase (PI3K)/protein kinase B (Akt) signaling pathways. The increase in NO levels may, in turn, enhance SIRT1 activity, which, once more, may delay endothelial senescence [59]. Summarizing, during ageing, oxidative stress accumulates, paralleled with a decrease in NO production, which might be responsible for SIRT1 inactivation. This negative loop facilitates the senescence-like phenotype of endothelial cells (Figure 2). Indeed, it has been recently demonstrated that statins, which induce eNOS activity via SIRT1 upregulation, may inhibit oxidative-dependent endothelial senescence [60].

Figure 2. Oxidative stress, epigenetics and diseases. iNOS, inducible nitric oxide synthase; eNOS, endothelial nitric oxide synthase.

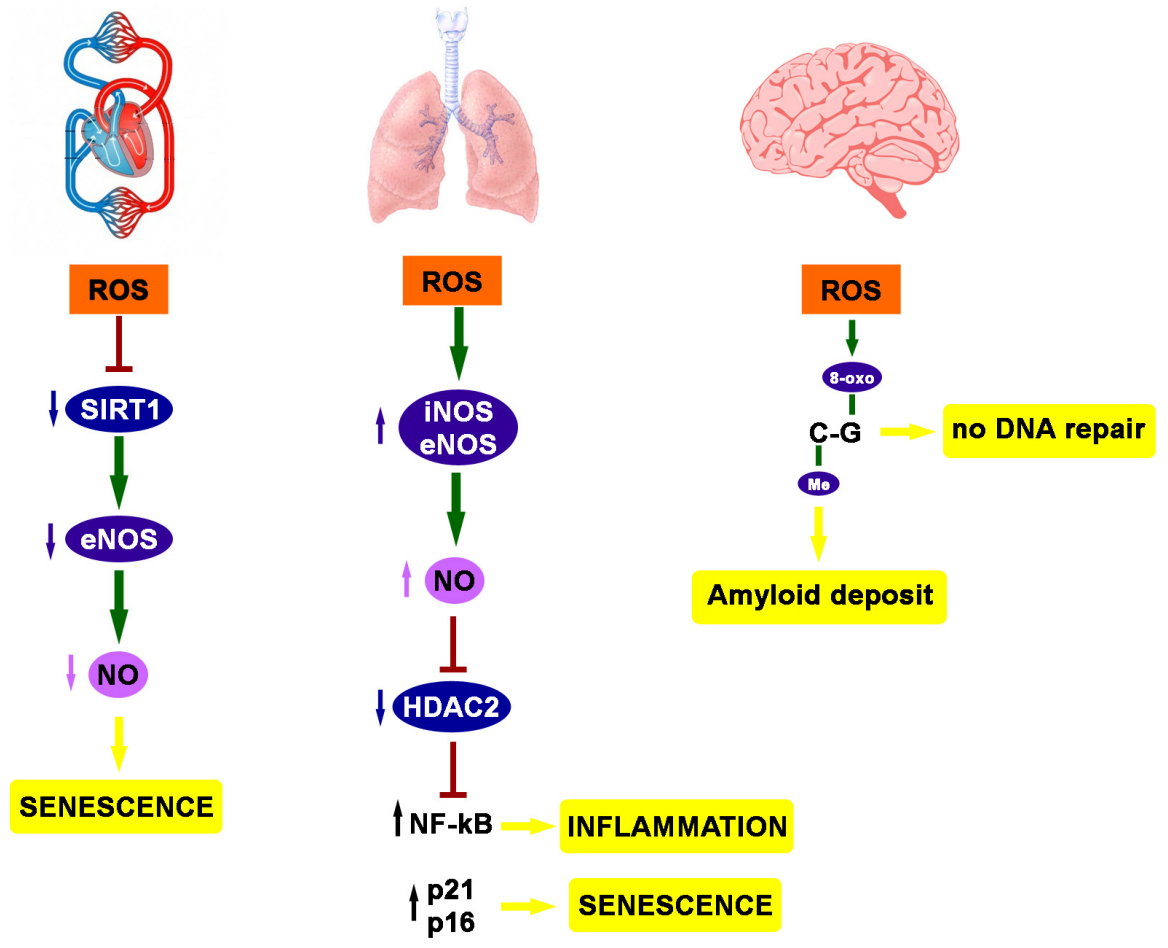

Besides the cardiovascular system, lungs are exposed to either endogenous or exogenous sources of oxidants. The endogenous oxidants predominantly derive from mitochondrial respiration and phagocyte activation, whereas important exogenous determinants of oxidation are air pollutants, noxious gases and, last, but not least, the smoke of cigarettes [61,62]. The accumulation of ROS directly impairs the function of lung cells, determining posttranslational modifications of histones and non-histone proteins, as well as that of chromatin remodeling enzymes [61,62]. The lung disease in which all these mechanisms are the most evident is chronic obstructive pulmonary disease (COPD) 
characterized by chronic low-grade systemic inflammation and premature ageing, the so-called "inflamm-ageing", which determines the obstruction of lung airflow and decreases respiratory function [63]. In these patients, inflammation and cellular senescence are exacerbated by tobacco smoke, which accelerates or induces premature lung ageing [63-65]. Indeed, cigarette smoke contains a number of free radicals and chemical compounds, representing the major source of inhaled ROS, able to alter the intracellular balance between acetylation/deacetylation and methylation/demethylation processes, leading to a deregulated expression of proinflammatory genes [64,65]. Specifically, it has been recently found that cigarette smoke post-translationally modifies histone deacetylase 2 (HDAC2), a class I histone deacetylase, causing a significant reduction in its enzymatic activity [64]. Adenuga et al. [66] observed a smoke-dependent HDAC2 inactivation by phosphorylation at Ser394, Ser411, Ser422 and Ser424 in macrophages, human bronchial and primary small airway lung epithelial cells and, in vivo, in the mouse lung. In this context, it is the caseine protein kinase 2 (CK2) that induces HDAC2 phosphorylation, leading to its inactivation by ubiquitination and degradation via the proteasome pathway. This physiopathological condition is associated with severe unfavorable effects, such as steroid resistance and abnormal inflammation [66]. Besides phosphorylation, HDAC2 can be modified and inactivated by smoke-induced carbonyl stress and NO-dependent $S$-nitrosylation at cysteine [67,68]. Indeed, in the mouse lung, tobacco smoke increases inducible NOS (iNOS) and eNOS expression and function, respectively, with a consequent increment of NO generation [69]. In this regard, we reported that a deregulated NO synthesis in mice expressing a constitutively active form of eNOS leads to $S$-nitrosylation of HDAC2, with a subsequent loss of its deacetylase activity [70]. A decreased HDAC2 activity has been associated with inflammation and senescence in COPD patients via the increase of histones $\mathrm{H} 3$ and $\mathrm{H} 4$ acetylation, the activation of the transcription factor, nuclear factor of kappa light polypeptide gene enhancer in B-cells 1 (NF- $\kappa B)$, and the unscheduled transcription of proinflammatory genes [66,71,72]. Moreover, HDAC2 activity normally delays cellular senescence by negatively regulating pro-senescent genes, such as cyclin-dependent kinase inhibitor $1 A(p 21)$ and cyclin-dependent kinase inhibitor $2 A$ (p16) [64]. Therefore, a significant reduction in HDAC2 function may accelerate cellular senescence and pulmonary emphysema in COPD patients (Figure 2).

Similarly to the cardiovascular and respiratory systems, the nervous system is also vulnerable to oxidative stress. In fact, although brain holds high concentrations of lipids susceptible to peroxidation and uses high amounts of oxygen to produce energy, it has a relatively deficient anti-oxidant system [73]. Indeed, several lines of evidence have recently underlined the role of oxidative stress and the simultaneous downregulation of antioxidant enzymes during progression from healthy ageing to dementia [74]. Alzheimer's disease, the typical dementia form of aged people, is characterized by progressive loss of memory and cognitive capacities, due to extracellular amyloid deposits, the so-called senile plaques, and to the formation of intraneuronal aggregates of hyper-phosphorylated tau protein, forming the so-called "neurofibrillary tangles" [74]. Oxidative DNA damage is now accepted as one of the earliest observable events in Alzheimer's pathogenesis. Remarkably, it can be detected in brains and in peripheral tissues of patients either affected by mild cognitive impairment or at their late stages of Alzheimer's disease [74]. The most frequent oxidative DNA lesion is the oxidation of guanine to 8-oxo-7,8-dihydro-2'-deoxyguanosine (8-oxo-G), which alters transcription factors binding to DNA as a consequence of a deranged epigenetic signaling [75-77]. Furthermore, astrocytes belonging to the hippocampus and cerebral cortex of Alzheimer's disease patients often present histone 
H2A member X phosphorylation, a hallmark of DNA double-strand breaks, which allows formation of intranuclear $\mathrm{y}-\mathrm{H} 2 \mathrm{AX}$ foci [78]. Of interest, a Cytosine nucleotide next to a Guanine nucleotide arrayed in a linear sequence forms the so-called "CpG island" in specific DNA regions, often intergenic and associated with gene expression control. In this context the presence of oxidized guanosine to form 8-oxo-G, which is one of the most common oxidative DNA damage biomarkers, is often associated with cytosine methylation, leading to the formation of methylated and oxidized CG stretches. These regions might represent sites of interplay between epigenetic and oxidative stress signals potentially relevant in Alzheimer's disease physiopathology, as proposed by Zawia and coworkers [79]. These authors found that external stimuli during rat brain development might reduce DNA-methyltransferase activity, leading to hypomethylation in the regulatory regions of genes associated with Alzheimer's disease, such as $\beta$-amyloid-precursor-proteins and secretases [79]. In this light, early life exposure to specific stimuli, such as xenobiotic metals, gives an impulse to Alzheimer's disease, inducing a progressive accumulation of $\beta$-amyloid-precursor-proteins and $\beta$-amyloids [79]. Coincidently to the formation of these deposits, an increase of cerebral 8-oxo-G levels has been observed [79]. In this way, the epigenetic imprinting can influence the expression of Alzheimer's disease-related genes, promoting DNA damage and pathogenesis progression. Remarkably, it has been observed that 8-oxo-G cannot be repaired when it is preceded by a methylcytosine [79]. Thus, in the presence of cytosines methylated early in life and belonging to $\mathrm{CpG}$ islands, the correction of adjacent guanines in the case of an oxidation event occurring late in life will be prevented, leading to accumulation of oxidative DNA damage in ageing brains (Figure 2) [79]. In conclusion, the authors established that methylation imprinting hits both gene expression and susceptibility to oxidative DNA damage in the late stages of Alzheimer's disease. Hence, the epigenetic machinery may represent an oxidative stress sensor that orchestrates the progressive homeostasis impairment typical of ageing, thus shaping the cellular senescence often observed during cardiovascular, respiratory and nervous system degeneration.

\section{Youth Fountain: Struggle with ROS}

Lifespan is often correlated to metabolic rate. Albeit that several exceptions exist, it is often observed that the faster the metabolism, the higher the ROS production and, thus, the shorter the lifespan. For this reason, the controlled reduction of oxidative stress may represent a way to slow the progressive homeostasis impairment occurring during ageing. At present, several studies pointed out different methods to increase organism lifespans, including caloric restriction, deletion of p66ShcA and enhancement of SIRT1 activity. All these methods have in common the ability to decrease oxidative stress [10].

Restricted caloric intake significantly modifies the rate of ageing and reduces the age-associated accumulation of oxidized damaged macromolecules [10]. Gene profiles of caloric-restricted aged mice shows low level expression of genes involved in oxidative stress in comparison with aged mice fed ad libitum $[6,10]$. Thus, caloric restriction prevents several gene expression changes usually occurring in age-related diseases, concurring to prolong animal lifespans by about $20 \%[10,80]$.

Sirtuins are the epigenetic beneficial effectors of caloric restriction [81,82]. They belong to a family of seven $\mathrm{NAD}^{+}$-dependent class III deacetylases, namely SIRT1-7 [83]. The most characterized component of the family is SIRT1. The aforementioned seems to protect against cardiovascular 
function impairment common in later ages, cope with stress stimuli and contribute to maintain telomere stability [81,82]. Oxidative stress, in fact, decreases SIRT1 activity to such an extent that some of the negative regulators of oxidative stress, such as p53, forkhead box, sub-group O 3 (FOXO3) and eNOS, become deacetylated and unable to efficiently counteract the progressive homeostasis impairment of endothelial cells [56]. Noteworthy, SIRT1 regulates NO production, contributing indirectly to vascular homeostasis [84]. Specifically, SIRT1 deacetylases eNOS on Lys496 and Lys506 and stimulates its activity [85]. Actually, during ageing, eNOS phosphorylation levels drop, whereas acetylation levels increase. Remarkably, the SIRT1-eNOS coupling not only improves endothelium-dependent vasomotor tone $[85,86]$, but possibly that of a larger number of cell types, as we recently demonstrated in keratinocytes during the skin repair process of mice [87].

Other molecules may have a negative effect on animal lifespan, as in the case of the p66 Src homology 2 domain-containing (p66ShcA) gene, whose deletion extends life in mice of by least 30\% [88]. p66ShcA, together with p46ShcA and p52ShcA, is one of the three isoforms of the mammalian adapter protein, ShcA [89]. All ShcA isoforms contain a common structure, but only p66ShcA presents a unique domain at the $\mathrm{N}$-terminus. p52 and p46 are cytoplasmic signal transduction molecules involved in mitogenic signaling from activated tyrosine kinase receptors to Ras, whereas the p66 isoform is devoid of this function and regulates ROS metabolism at the mitochondrial level, promoting oxidative stress in cells and tissues and apoptosis [89]. Epigenetics plays a pivotal role in the regulation of p66ShcA expression [90]. Indeed, p66ShcA expression is partially controlled by different epigenetic modifications of its promoter, which present a high content of CG nucleotides, although not sufficient to qualify as a $\mathrm{CpG}$ island-rich region [90]. As previously discussed, DNA methylation of CpG islands is a well-known gene silencing mechanism that confers high stability to chromatin and poor accessibility to transcriptional complexes. p66ShcA promoter analysis in different cell lines showed, in fact, a strong correlation between nucleotides methylation and the expression level of p66ShcA [90]. In cell lines expressing high levels of p66ShcA, bisulfite analysis showed that all the CpG were unmethylated [90]. Conversely, among cell lines not expressing detectable amounts of p66ShcA, the fraction of methylated cytosines ranged between $41 \%$ and $100 \%$. In support of DNA methylation as a silencer mechanism for the p66ShcA locus, Ventura and co-workers [90] demonstrated that demethylating treatment of these cell lines induces de novo transcription of the p66ShcA gene. In addition, further analyses revealed that p66ShcA is transcriptionally repressed by SIRT1, as confirmed by the evidence that p66Shc increases following SIRT1 inhibition. Specifically, SIRT1 directly regulates the p66Shc promoter, decreasing the acetylation of its histone, H3 [91].

Oxidative stress is a determinant of ageing, and p66ShcA knockout results in oxidative stress resistance and low levels of apoptosis. Indeed, murine embryonic fibroblasts derived from p66ShcA knockout (KO) mice are resistant to treatment with oxidant agents and only infrequently respond to oxidative stress stimuli, undergoing apoptosis [88]. On the contrary, murine embryonic fibroblasts overexpressing p66ShcA present an increased level of apoptosis, which correlates with the intracellular production of ROS [88]. In this context, we recently demonstrated that p66ShcA deletion increased both skeletal muscle and endothelial cell resistance to acute ischemia, a tissue injury in which the rapid formation of ROS plays a detrimental role [92]. Intriguingly, we found that p66ShcA not only modulated cell survival, but also differentiation of skeletal muscle progenitors and skeletal muscle regeneration after hind limb ischemia [93]. Moreover, as concerns diabetic injury, in which oxidative 
stress plays a pivotal role, we reported that the ability of p66ShcA to generate ROS was important for hyperglycemia-sensitivity in bone marrow-derived endothelial progenitor cells and that an active p66ShcA was responsible for the angiogenic impairment induced by diabetes in a mouse model of angiogenesis [94]. In line with this, Chen and coworkers observed the involvement of SIRT1 in diabetic mice [91]. Specifically, SIRT1 was downregulated in the aorta of diabetic mice, and this, in turn, triggered the activation of p66ShcA, causing hyperglycemia-induced endothelial dysfunction [91].

All this evidence shows that p66ShcA may function as a sensor of intracellular concentration of ROS, regulating apoptosis and lifespan. It is well established now that the absence of p66ShcA confers oxidative stress resistance and increases longevity, although this advantage may be limited to the laboratory environment in which animals are kept [95].

\section{Epigenetic Drugs in Ageing and Age-Related Diseases}

Besides genetic interventions, the promise of "healthy ageing" can be pursued, developing epigenetics drugs able to cope with the "aged epigenome".

The increase of SIRT1 expression and/or activity has positive effects in type 2 diabetes, cancer, cardiovascular diseases, COPD and Alzheimer's disease [96]. In this light, sirtuin therapeutic activation, by small molecules, is thought to provide a new approach to treat or prevent age-related diseases.

Since 2003, resveratrol (see 1 in Figure 3) has been identified as a potent SIRT1 activator that mimics the effect of caloric restriction and regulates longevity in yeast, worms, flies, short-lived fish and mice [96]. In obese rodents, treatment with resveratrol produces a variety of health benefits, including improved metabolic and vascular function, decreased hepatic steatosis, reduced inflammation and improved endurance. Recent clinical studies showed that resveratrol also confers metabolic benefits to humans. In obese humans, one month of resveratrol supplementation, in fact, induced metabolic changes, mimicking the effect of caloric restriction. This beneficial effect has been associated with the positive effect of resveratrol on SIRT1 and the consequent reduction of cellular senescence and inflammation [97]. Resveratrol is currently being evaluated in clinical trials for the treatment of several ageing-related pathologies (see Table 2 for details).

Other epigenetic molecules are now under evaluation for their potentially positive effect in age-associated diseases. Quercetin (2), in fact, has been shown to protect against emphysema, a beneficial effect probably due to an increased expression of SIRT1. This observation is in agreement with prior studies about the property of quercetin to activate mammalian SIRT1 or its yeast orthologous Sir2 [98]. Other polyphenolic compounds, including piceatannol (3), can also activate SIRT1. Although these compounds have a modest effect on SIRT1, compared to resveratrol, nevertheless, they may have a beneficial application in the treatment of lung inflammation [98].

Several synthetic SIRT1 activators have been recently developed for the treatment of age-associated diseases, including type 2 diabetes [98]. These activators are known as SRT1720 (4), SRT1460 (5), SRT2183 (6), SRT2104 and SRT2379. The most potent among these compounds is SRT1720 $\left(\mathrm{EC}_{1.5}=0.16 \mu \mathrm{M}\right)$, which improves glucose homeostasis and insulin sensitivity in animal models of type 2 diabetes [99]. Furthermore, due to SIRT1 activation, it was found that SRT1720 reduced cigarette smoke-induced cellular senescence in the lung. [100]. In addition, SRT1720 improved survival and the health of obese mice [101], suggesting that designing novel molecules that are safe 
and effective in promoting longevity and preventing multiple age-related diseases in mammals may represent a promising perspective. Of note, some of these compounds are now in phase I/II clinical trials (see Table 2).

Figure 3. Epigenetic small molecule modulators in ageing and age-related diseases.<smiles>Oc1ccc(/C=C/c2cc(O)cc(O)c2)cc1</smiles>

resveratrol (1)<smiles>O=C1c2c(O)cc(O)cc2C=C(c2ccc(O)c(O)c2)C1O</smiles>

quercetin (2)<smiles>Oc1cc(O)cc(C=Cc2ccc(O)c(O)c2)c1</smiles>

piceatannol (3)<smiles>O=C(Nc1cccc(-c2cn3c(CN4CCNCC4)csc3n2)c1)c1cnc2ccccc2n1</smiles><smiles>COc1cc(C(=O)Nc2ccccc2-c2cn3c(CN4CCNCC4)csc3n2)cc(OC)c1O</smiles><smiles></smiles><smiles>CC(=O)C1=CN(Cc2ccccc2)C=C(C(C)=O)C1c1ccccc1</smiles><smiles>CN(C)C(=N)NC(=N)N</smiles>
metformin (8)<smiles>O=C1CCc2cc(OCCCCc3nnnn3C3CCCCC3)ccc2N1</smiles><smiles>C[14CH3]</smiles><smiles>NC(=O)CCCc1ccccc1</smiles><smiles>CC(=C/[C@@H](C)C(=O)c1ccc(N(C)C)cc1)/C=C/C(=O)NO</smiles><smiles>CC(=O)N[C@@H](CCCCNC(=O)CO)C(N)=O</smiles>
sodium 4-phenylbutyrate (11)

$\mathrm{CH}_{3} \quad$ trichostatin A, TSA (12)<smiles>CC(C)(C)CCCCN1C(=O)c2cccc3cccc(c23)C1=O</smiles>
Lys-CoA (10)<smiles>Nc1ccccc1NC(=O)c1ccc(CNc2nccc(-c3cccnc3)n2)cc1</smiles>

MGCD0103, mocetinostat (14)<smiles>CC(=O)c1ccc2c(c1)nc(NC(=O)c1ccccc1)n2CCCc1ccccc1</smiles>

BRD4770 (15)

Table 2. Epigenetic modulators in clinical trials for age-related diseases.

\begin{tabular}{cccc}
\hline Drugs & Condition & clinicaltrials.gov Identifier & Phase \\
\hline Resveratrol & Type 2 diabetes & NCT01677611 & I, Completed \\
Resveratrol & $\begin{array}{c}\text { Vascular resistance, aging, hypertension, } \\
\text { antioxidants, aerobic capacity }\end{array}$ & NCT01842399 & II \\
Resveratrol & Healthy & NCT00996229 & III \\
Resveratrol & Alzheimer's disease & NCT00678431 & III, completed \\
SRT-2104 & Type 2 diabetes & NCT00937872, NCT00933062, & I, II \\
SRT-2379 & Type 2 diabetes & NCT00933530, NCT01018017 & I \\
Metformin & COPD & NCT01018628 & IV \\
\hline
\end{tabular}


In 2009, a new class of 1,4-dihydropyridine derivatives (DHPs) was recognized as a novel SIRT activator $\left(\mathrm{EC}_{1.5}\right.$, SIRT1 $\left.=1 \mu \mathrm{M}\right)$, showing a reduction in cellular senescence of primary human mesenchymal stem cells similar to resveratrol. When tested in murine $\mathrm{C} 2 \mathrm{C} 12$ myoblast cell line, the most potent compound of this class, diethyl 1-benzyl-1,4-dihydro-4-phenylpyridine-3,5-dicarboxylatenamed (MC2562, 7), showed a dose-dependent increase in mitochondrial activity with a mechanism involving PGC-1 $\alpha$ [102]. In vitro and in vivo studies revealed that the activation of SIRTs by MC2562 stimulated keratinocyte proliferation via eNOS phosphorylation and NO production, highlighting its effectiveness in accelerating wound repair in a mouse experimental model of skin damage [87].

Metformin (8) is a widely used drug for the reduction of hyperglycemia in type 2 diabetes. A recent study demonstrated that the beneficial effect of metformin is associated with the activation and induction of SIRT1 [103]. Further studies revealed that metformin targets AMP-activated protein kinase (AMPK), an upstream kinase important for activating SIRT1. Although the mechanism of metformin action in diabetes, lung inflammation and other ageing-associated diseases remains elusive, clinical trials are ongoing to study the effect of metformin in the treatment of COPD and its complications (Table 2).

Cilostazol (9), a selective inhibitor of PDE3, has been reported to protect endothelium, after ischemic damage, through the induction of a significant production of NO [59]. It seems to increase eNOS phosphorylation via a dose-dependent positive effect on SIRT1 expression. The effect of cilostazol on premature senescence is, in fact, abrogated by SIRT1 inhibition [59].

Expression levels of the histone acetyltransferases, p300 and cAMP-responsive element-binding protein-binding protein (CBP), have been reported to decrease with age in mouse models [104]. Remarkably, the genetic or pharmacological inhibition of p300 activity [the latter obtained by using Lys-CoA (10), a bi-substrate p300 inhibitor] led to growth inhibition, downregulation of cyclin $\mathrm{E}$ and activation of the senescence-associated acidic $\beta$-galactosidase in human melanocytes and melanoma cells, whose proliferation often occurs in elderly people [105].

Although HDAC inhibitors (HDACi) are mainly studied for their anti-cancer activity, they also show other biological properties, including anti-inflammatory and neuroprotective ones. In recent years, experimental data emerged on the life-extending potential of synthetic HDACi. A substantial increase in both average and maximum survival without loss of motility, resistance to stress or fertility was observed during feeding Drosophila melanogaster with the HDACi, 4-phenylbutyrate (11), throughout adulthood [106]. Another study found that also trichostatin A (TSA, 12), the prototype pan-HDAC inhibitor, significantly extended the lifespan of flies [107]. Further experiments showed that both TSA and phenylbutyrate were to extend Drosophila lifespan [108].

In vivo studies demonstrated that pan-HDACi can slow or reverse pathological cardiac hypertrophy $[109,110]$. Treatment with the pan-HDACi TSA, in fact, reduced or prevented the development of cardiac hypertrophy in transgenic mice. TSA treatment was also shown to reverse established cardiac hypertrophy in mice subjected to aortic constriction. Another HDACi, scriptaid (13), has been found to be able to blunt cardiac hypertrophy in a pressure-overload mouse model, reducing the size of cardiomyocytes, while improving ventricular performance. In this context, studies performed in genetically engineered mice and isolated cardiomyocytes suggested a role for HDAC2 in heart failure. More definitive answers likely will come from the use of small molecule inhibitors tailored to selected HDAC isoforms. An apicidin derivative (API-D) which is selective predominantly for the class I HDACs, 1, 2 and 3, was shown to effectively suppress cardiac hypertrophy and to improve 
cardiac performance in the presence of pressure overload [110]. Recently, it has been reported that only the class I HDACi mocetinostat, (MGCD-0103, 14), and not a class II HDACi, was able to re-express the dual specificity protein phosphatase 5 (dusp5) gene, leading to the inhibition of pro-hypertrophic gene expression. This finding enlightens a potentially novel pathway target of HDACi [111].

A number of histone methyl markers have been reported to be modified with ageing. In general, in vitro and in vivo studies revealed a global increase in H4K20me3, as well as a decrease of tri-methylated lysine 9 of histone $\mathrm{H} 3$ (H3K9me3) and H3K27me3. Interestingly, the Ash-2 complex, which trimethylates $\mathrm{H} 3 \mathrm{~K} 4$, is a negative regulator of lifespan in Caenorhabditis elegans [31-35]. Nevertheless, only one small molecule, the 2-(Benzoylamino)-1-(3-phenylpropyl)-1H-benzimidazole5-carboxylic acid methyl ester (BRD4770) compound (15), has been recently described to inhibit the lysine 9 of histone H3 (H3K9) methyltransferase, G9a, reducing the levels of H3K9me3 and inducing senescence in pancreatic adenocarcinoma PANC-1 cells through activation of ataxia telangiectasia mutated (ATM) kinase [112]. In light of these observations, although the situation is promising, a large amount of work remains to be done in the field of epigenetics to develop effective and enzyme-specific drugs with potential therapeutic application in ageing-associated diseases.

\section{Concluding Remarks}

The accumulation of oxidative stress might orchestrate the progressive homeostasis impairment that leads to the loss of function typical of aged tissues, which often degenerate in severe pathologies, such as coronary artery diseases, Alzheimer's disease and COPD. Here, we reviewed how epigenetics, using all its "weapons" such as histone-modifying enzymes and DNA-methylation, rules out stress stimuli and identifies part of the mechanisms associated with the physiopathology of ageing-associated diseases. In summary, ageing presents specific epigenetic markers, which, taken altogether, could define the ageing epigenome. These modifications may also be part of a physiopathological processes undergone during the onset of ageing-associated diseases. The next challenge will be the manipulation of this modified epigenome by the use of small molecules: in fact, despite the evidence of a great number of epi-markers, which change during ageing, only a few epi-drugs have been tested in this context, so far. The understanding of epigenetic pathways involved in ageing and ageing-associated diseases cues the development of new therapeutic treatments to contrast relentless tissue impairment, thus promising "healthy ageing". In this context, we suggest that controlling ROS production may represent the first step towards the achievement of this aim. As described, SIRT1 and p66ShcA, strictly interconnected with each other, might represent two promising targets conferring oxidative stress resistance to target cells and, consequently, delaying organism functional impairment. Although quite a large amount of work is still needed and there is evidence that the above-mentioned targets are not the only ones important in gaining "healthy ageing", they may represent the beginning of the struggle to control ageing physiology and physiopathology.

\section{Acknowledgments}

This work was supported by the LOEWE Center for Cell and Gene Therapy (LOEWE-CGT Frankfurt to C.G.) - Territorial Initiative for Scientific and Economic Excellence and by LOEWE-CGT, Frankfurt, funding reference number: III L 4-518/17.004 (2010 to F.S.). This study was supported by 
Ministero della Salute and by AIRC IG11436 to F.M. Thanks are due to FIRB RBFR10ZJQT, Progetto IIT-Sapienza A2, FP7 Project BLUEPRINT/282510 and FP7 COST/TD0905 to S.V. and A.M.

\section{Conflicts of Interest}

The authors declare no conflict of interest.

\section{References}

1. Martin, G.M. The biology of aging: 1985-2010 and beyond. FASEB J. 2011, 25, 3756-3762.

2. Olshansky, S.J.; Goldman, D.P.; Zheng, Y.; Rowe, J.W. Aging in America in the twenty-first century: Demographic forecasts from the MacArthur foundation research network on an aging society. Milbank Q. 2009, 87, 842-862.

3. Hayflick, L. Biological aging is no longer an unsolved problem. Ann. N. Y. Acad. Sci. 2007, $1100,1-13$.

4. Kirkwood, T.B. Understanding the odd science of aging. Cell 2005, 120, 437-447.

5. Beckman, K.B.; Ames, B.N. The free radical theory of aging matures. Physiol. Rev. 1998, 78, $547-581$.

6. Lee, C.K.; Klopp, R.G.; Weindruch, R.; Prolla, T.A. Gene expression profile of aging and its retardation by caloric restriction. Science 1999, 285, 1390-1393.

7. Stadtman, E.R. Protein oxidation and aging. Science 1992, 257, 1220-1224.

8. Balaban, R.S.; Nemoto, S.; Finkel, T. Mitochondria, oxidants, and aging. Cell 2005, 120, 483-495.

9. Haigis, M.C.; Yankner, B.A. The aging stress response. Mol. Cell 2010, 40, 333-344.

10. Sohal, R.S.; Weindruch, R. Oxidative stress, caloric restriction, and aging. Science 1996, 273, 59-63.

11. Davies, K.J. Oxidative stress, antioxidant defenses, and damage removal, repair, and replacement systems. IUBMB Life 2000, 50, 279-289.

12. Finkel, T.; Holbrook, N.J. Oxidants, oxidative stress and the biology of ageing. Nature 2000, 408, 239-247.

13. Stadtman, E.R. Protein oxidation in aging and age-related diseases. Ann. N. Y. Acad. Sci. 2001, 928, 22-38.

14. Ben-Avraham, D.; Muzumdar, R.H.; Atzmon, G. Epigenetic genome-wide association methylation in aging and longevity. Epigenomics 2012, 4, 503-509.

15. Berger, S.L.; Kouzarides, T.; Shiekhattar, R.; Shilatifard, A. An operational definition of epigenetics. Genes Dev. 2009, 23, 781-783.

16. Skinner, M.K. Environmental epigenetic transgenerational inheritance and somatic epigenetic mitotic stability. Epigenetics 2011, 6, 838-842.

17. Illi, B.; Colussi, C.; Grasselli, A.; Farsetti, A.; Capogrossi, M.C.; Gaetano, C. NO sparks off chromatin: Tales of a multifaceted epigenetic regulator. Pharmacol. Ther. 2009, 123, 344-352.

18. Williams, K.; Christensen, J.; Helin, K. DNA methylation: TET proteins-guardians of CpG islands? EMBO Rep. 2011, 13, 28-35.

19. Cedar, H.; Bergman, Y. Programming of DNA methylation patterns. Annu. Rev. Biochem. 2012, 81, 97-117.

20. Gronbaek, K.; Hother, C.; Jones, P.A. Epigenetic changes in cancer. APMIS 2007, 115, 1039-1059. 
21. Guil, S.; Esteller, M. Cis-acting noncoding RNAs: Friends and foes. Nat. Struct. Mol. Biol. 2011, 19, 1068-1075.

22. Vanyushin, B.F.; Nemirovsky, L.E.; Klimenko, V.V.; Vasiliev, V.K.; Belozersky, A.N. The 5-methylcytosine in DNA of rats. Tissue and age specificity and the changes induced by hydrocortisone and other agents. Gerontologia 1973, 19, 138-152.

23. Choi, E.K.; Uyeno, S.; Nishida, N.; Okumoto, T.; Fujimura, S.; Aoki, Y.; Nata, M.; Sagisaka, K.; Fukuda, Y.; Nakao, K.; et al. Alterations of $c$-fos gene methylation in the processes of aging and tumorigenesis in human liver. Mutat. Res. 1996, 354, 123-128.

24. Issa, J.P.; Vertino, P.M.; Boehm, C.D.; Newsham, I.F.; Baylin, S.B. Switch from monoallelic to biallelic human IGF2 promoter methylation during aging and carcinogenesis. Proc. Natl. Acad. Sci. USA 1996, 93, 11757-11762.

25. So, K.; Tamura, G.; Honda, T.; Homma, N.; Waki, T.; Togawa, N.; Nishizuka, S.; Motoyama, T. Multiple tumor suppressor genes are increasingly methylated with age in non-neoplastic gastric epithelia. Cancer Sci. 2006, 97, 1155-1158.

26. Gonzalo, S. Epigenetic alterations in aging. J. Appl. Physiol. 2010, 109, 586-597.

27. Pruitt, K.; Zinn, R.L.; Ohm, J.E.; McGarvey, K.M.; Kang, S.H.; Watkins, D.N.; Herman, J.G.; Baylin, S.B. Inhibition of SIRT1 reactivates silenced cancer genes without loss of promoter DNA hypermethylation. PLoS Genet. 2006, 2, e40.

28. Vaquero, A.; Scher, M.; Erdjument-Bromage, H.; Tempst, P.; Serrano, L.; Reinberg, D. SIRT1 regulates the histone methyl-transferase SUV39H1 during heterochromatin formation. Nature 2007, 450, 440-444.

29. Thakur, M.K.; Kanungo, M.S. Methylation of chromosomal proteins and DNA of rat brain and its modulation by estradiol and calcium during aging. Exp. Gerontol. 1981, 16, 331-336.

30. Wang, C.M.; Tsai, S.N.; Yew, T.W.; Kwan, Y.W.; Ngai, S.M. Identification of histone methylation multiplicities patterns in the brain of senescence-accelerated prone mouse 8 . Biogerontology 2010, 11, 87-102.

31. Fraga, M.F.; Esteller, M. Epigenetics and aging: The targets and the marks. Trends Genet. 2007, 23, 413-418.

32. Sarg, B.; Koutzamani, E.; Helliger, W.; Rundquist, I.; Lindner, H.H. Postsynthetic trimethylation of histone $\mathrm{H} 4$ at lysine 20 in mammalian tissues is associated with aging. J. Biol. Chem. 2002, 277, 39195-39201.

33. Agger, K.; Cloos, P.A.; Rudkjaer, L.; Williams, K.; Andersen, G.; Christensen, J.; Helin, K. The H3K27me3 demethylase JMJD3 contributes to the activation of the INK4A-ARF locus in response to oncogene- and stress-induced senescence. Genes Dev. 2009, 23, 1171-1176.

34. Jung, J.W.; Lee, S.; Seo, M.S.; Park, S.B.; Kurtz, A.; Kang, S.K.; Kang, K.S. Histone deacetylase controls adult stem cell aging by balancing the expression of polycomb genes and jumonji domain containing 3. Cell. Mol. Life Sci. 2010, 67, 1165-1176.

35. Narita, M.; Nunez, S.; Heard, E.; Narita, M.; Lin, A.W.; Hearn, S.A.; Spector, D.L.; Hannon, G.J.; Lowe, S.W. Rb-mediated heterochromatin formation and silencing of $E 2 F$ target genes during cellular senescence. Cell 2003, 113, 703-716.

36. Zhang, R.; Adams, P.D. Heterochromatin and its relationship to cell senescence and cancer therapy. Cell Cycle 2007, 6, 784-789. 
37. Ye, X.; Zerlanko, B.; Zhang, R.; Somaiah, N.; Lipinski, M.; Salomoni, P.; Adams, P.D. Definition of pRB- and p53-dependent and -independent steps in HIRA/ASF1a-mediated formation of senescence-associated heterochromatin foci. Mol. Cell. Biol. 2007, 27, 2452-2465.

38. Boulias, K.; Horvitz, H.R. The C. elegans microRNA mir-71 acts in neurons to promote germline-mediated longevity through regulation of DAF-16/FOXO. Cell Metab. 2012, 15, 439-450.

39. Boon, R.A.; Seeger, T.; Heydt, S.; Fischer, A.; Hergenreider, E.; Horrevoets, A.J.; Vinciguerra, M.; Rosenthal, N.; Sciacca, S.; Pilato, M.; et al. MicroRNA-29 in aortic dilation: Implications for aneurysm formation. Circ. Res. 2011, 109, 1115-1119.

40. Menghini, R.; Casagrande, V.; Cardellini, M.; Martelli, E.; Terrinoni, A.; Amati, F.; Vasa-Nicotera, M.; Ippoliti, A.; Novelli, G.; Melino, G.; et al. MicroRNA 217 modulates endothelial cell senescence via silent information regulator 1. Circulation 2009, 120, 1524-1532.

41. Olivieri, F.; Lazzarini, R.; Recchioni, R.; Marcheselli, F.; Rippo, M.R.; di Nuzzo, S.; Albertini, M.C.; Graciotti, L.; Babini, L.; Mariotti, S.; et al. MiR-146a as marker of senescence-associated pro-inflammatory status in cells involved in vascular remodelling. Age 2012, 35, 1157-1172.

42. Boon, R.A.; Iekushi, K.; Lechner, S.; Seeger, T.; Fischer, A.; Heydt, S.; Kaluza, D.; Treguer, K.; Carmona, G.; Bonauer, A.; et al. MicroRNA-34a regulates cardiac ageing and function. Nature 2013, 495, 107-110.

43. Ito, T.; Yagi, S.; Yamakuchi, M. MicroRNA-34a regulation of endothelial senescence. Biochem. Biophys. Res. Commun. 2010, 398, 735-740.

44. Magenta, A.; Cencioni, C.; Fasanaro, P.; Zaccagnini, G.; Greco, S.; Sarra-Ferraris, G.; Antonini, A.; Martelli, F.; Capogrossi, M.C. miR-200c is upregulated by oxidative stress and induces endothelial cell apoptosis and senescence via ZEB1 inhibition. Cell Death Differ. 2011, $18,1628-1639$.

45. Kenyon, C.; Chang, J.; Gensch, E.; Rudner, A.; Tabtiang, R. A C. elegans mutant that lives twice as long as wild type. Nature 1993, 366, 461-464.

46. Sedding, D.G. FoxO transcription factors in oxidative stress response and ageing-A new fork on the way to longevity? Biol. Chem. 2008, 389, 279-283.

47. Kaeberlein, M.; McVey, M.; Guarente, L. The SIR2/3/4 complex and SIR2 alone promote longevity in Saccharomyces cerevisiae by two different mechanisms. Genes Dev. 1999, 13, 2570-2580.

48. Romanov, V.S.; Pospelov, V.A.; Pospelova, T.V. Cyclin-dependent kinase inhibitor p21 ${ }^{\text {Waf1. }}$ Contemporary view on its role in senescence and oncogenesis. Biochemistry (Mosc.) 2012, 77, 575-584.

49. Bhaumik, D.; Scott, G.K.; Schokrpur, S.; Patil, C.K.; Orjalo, A.V.; Rodier, F.; Lithgow, G.J.; Campisi, J. MicroRNAs miR-146a/b negatively modulate the senescence-associated inflammatory mediators IL-6 and IL-8. Aging (Albany N.Y.) 2009, 1, 402-411.

50. Vasa-Nicotera, M.; Chen, H.; Tucci, P.; Yang, A.L.; Saintigny, G.; Menghini, R.; Mahe, C.; Agostini, M.; Knight, R.A.; Melino, G.; et al. miR-146a is modulated in human endothelial cell with aging. Atherosclerosis 2011, 217, 326-330.

51. North, B.J.; Sinclair, D.A. The intersection between aging and cardiovascular disease. Circ. Res. 2012, 110, 1097-1108. 
52. Oxenham, H.; Sharpe, N. Cardiovascular aging and heart failure. Eur. J. Heart Fail. 2003, 5, 427-434.

53. Versari, D.; Daghini, E.; Virdis, A.; Ghiadoni, L.; Taddei, S. The ageing endothelium, cardiovascular risk and disease in man. Exp. Physiol. 2009, 94, 317-321.

54. Strait, J.B.; Lakatta, E.G. Aging-associated cardiovascular changes and their relationship to heart failure. Heart Fail. Clin. 2012, 8, 143-164.

55. Tsutsui, M.; Shimokawa, H.; Morishita, T.; Nakashima, Y.; Yanagihara, N. Development of genetically engineered mice lacking all three nitric oxide synthases. J. Pharmacol. Sci. 2006, $102,147-154$.

56. Hwang, J.W.; Yao, H.; Caito, S.; Sundar, I.K.; Rahman, I. Redox regulation of SIRT1 in inflammation and cellular senescence. Free Radic. Biol. Med. 2013, 61C, 95-110.

57. Ota, H.; Tokunaga, E.; Chang, K.; Hikasa, M.; Iijima, K.; Eto, M.; Kozaki, K.; Akishita, M.; Ouchi, Y.; Kaneki, M. SIRT1 inhibitor, Sirtinol, induces senescence-like growth arrest with attenuated Ras-MAPK signaling in human cancer cells. Oncogene 2006, 25, 176-185.

58. Ota, H.; Akishita, M.; Eto, M.; Iijima, K.; Kaneki, M.; Ouchi, Y. SIRT1 modulates premature senescence-like phenotype in human endothelial cells. J. Mol. Cell. Cardiol. 2007, 43, 571-579.

59. Ota, H.; Eto, M.; Kano, M.R.; Ogawa, S.; Iijima, K.; Akishita, M.; Ouchi, Y. Cilostazol inhibits oxidative stress-induced premature senescence via upregulation of SIRT1 in human endothelial cells. Arterioscler. Thromb. Vasc. Biol. 2008, 28, 1634-1639.

60. Ota, H.; Eto, M.; Kano, M.R.; Kahyo, T.; Setou, M.; Ogawa, S.; Iijima, K.; Akishita, M.; Ouchi, Y. Induction of endothelial nitric oxide synthase, SIRT1, and catalase by statins inhibits endothelial senescence through the Akt pathway. Arterioscler. Thromb. Vasc. Biol. 2010, 30, 2205-2211.

61. Rahman, I.; Marwick, J.; Kirkham, P. Redox modulation of chromatin remodeling: Impact on histone acetylation and deacetylation, NF-kappaB and pro-inflammatory gene expression. Biochem. Pharmacol. 2004, 68, 1255-1267.

62. Repine, J.E.; Bast, A.; Lankhorst, I. The oxidative stress study group. Oxidative stress in chronic obstructive pulmonary disease. Am. J. Respir. Crit. Care Med. 1997, 156, 341-357.

63. Tuder, R.M.; Kern, J.A.; Miller, Y.E. Senescence in chronic obstructive pulmonary disease. Proc. Am. Thorac. Soc. 2012, 9, 62-63.

64. Yao, H.; Rahman, I. Role of histone deacetylase 2 in epigenetics and cellular senescence: Implications in lung inflammaging and COPD. Am. J. Physiol. Lung Cell. Mol. Physiol. 2012, 303, L557-L566.

65. Sundar, I.K.; Yao, H.; Rahman, I. Oxidative stress and chromatin remodeling in chronic obstructive pulmonary disease and smoking-related diseases. Antioxid. Redox. Signal. 2012, 18, 1956-1971.

66. Adenuga, D.; Yao, H.; March, T.H.; Seagrave, J.; Rahman, I. Histone deacetylase 2 is phosphorylated, ubiquitinated, and degraded by cigarette smoke. Am. J. Respir. Cell. Mol. Biol. 2009, 40, 464-473.

67. Marwick, J.A.; Kirkham, P.A.; Stevenson, C.S.; Danahay, H.; Giddings, J.; Butler, K.; Donaldson, K.; Macnee, W.; Rahman, I. Cigarette smoke alters chromatin remodeling and induces proinflammatory genes in rat lungs. Am. J. Respir. Cell. Mol. Biol. 2004, 31, 633-642. 
68. Moodie, F.M.; Marwick, J.A.; Anderson, C.S.; Szulakowski, P.; Biswas, S.K.; Bauter, M.R.; Kilty, I.; Rahman, I. Oxidative stress and cigarette smoke alter chromatin remodeling but differentially regulate $\mathrm{NF}-\kappa \mathrm{B}$ activation and proinflammatory cytokine release in alveolar epithelial cells. Faseb. J. 2004, 18, 1897-1899.

69. Seimetz, M.; Parajuli, N.; Pichl, A.; Veit, F.; Kwapiszewska, G.; Weisel, F.C.; Milger, K.; Egemnazarov, B.; Turowska, A.; Fuchs, B.; et al. Inducible NOS inhibition reverses tobacco-smoke-induced emphysema and pulmonary hypertension in mice. Cell 2011, 147, 293-305.

70. Colussi, C.; Mozzetta, C.; Gurtner, A.; Illi, B.; Rosati, J.; Straino, S.; Ragone, G.; Pescatori, M.; Zaccagnini, G.; Antonini, A.; et al. HDAC2 blockade by nitric oxide and histone deacetylase inhibitors reveals a common target in Duchenne muscular dystrophy treatment. Proc. Natl. Acad. Sci. USA 2008, 105, 19183-19187.

71. Rajendrasozhan, S.; Yang, S.R.; Kinnula, V.L.; Rahman, I. SIRT1, an antiinflammatory and antiaging protein, is decreased in lungs of patients with chronic obstructive pulmonary disease. Am. J. Respir. Crit. Care Med. 2008, 177, 861-870.

72. Yang, S.R.; Chida, A.S.; Bauter, M.R.; Shafiq, N.; Seweryniak, K.; Maggirwar, S.B.; Kilty, I.; Rahman, I. Cigarette smoke induces proinflammatory cytokine release by activation of NF- $\mathrm{kB}$ and posttranslational modifications of histone deacetylase in macrophages. Am. J. Physiol. Lung Cell. Mol. Physiol. 2006, 291, L46-L57.

73. Kedar, N.P. Can we prevent Parkinson's and Alzheimer's disease? J. Postgrad. Med. 2003, 49, 236-245.

74. Coppede, F.; Migliore, L. Evidence linking genetics, environment, and epigenetics to impaired DNA repair in Alzheimer's disease. J. Alzheimers Dis. 2010, 20, 953-966.

75. Dizdaroglu, M.; Jaruga, P.; Birincioglu, M.; Rodriguez, H. Free radical-induced damage to DNA: Mechanisms and measurement. Free Radic. Biol. Med. 2002, 32, 1102-1115.

76. Einolf, H.J.; Schnetz-Boutaud, N.; Guengerich, F.P. Steady-state and pre-steady-state kinetic analysis of 8-oxo-7,8-dihydroguanosine triphosphate incorporation and extension by replicative and repair DNA polymerases. Biochemistry 1998, 37, 13300-13312.

77. Hayakawa, H.; Taketomi, A.; Sakumi, K.; Kuwano, M.; Sekiguchi, M. Generation and elimination of 8-oxo-7,8-dihydro-2'-deoxyguanosine 5'-triphosphate, a mutagenic substrate for DNA synthesis, in human cells. Biochemistry 1995, 34, 89-95.

78. Myung, N.H.; Zhu, X.; Kruman, I.I.; Castellani, R.J.; Petersen, R.B.; Siedlak, S.L.; Perry, G.; Smith, M.A.; Lee, H.G. Evidence of DNA damage in Alzheimer disease: Phosphorylation of histone H2AX in astrocytes. Age 2008, 30, 209-215.

79. Zawia, N.H.; Lahiri, D.K.; Cardozo-Pelaez, F. Epigenetics, oxidative stress, and Alzheimer disease. Free Radic. Biol. Med. 2009, 46, 1241-1249.

80. Masoro, E.J. Caloric restriction and aging: An update. Exp. Gerontol. 2000, 35, 299-305.

81. Nemoto, S.; Fergusson, M.M.; Finkel, T. Nutrient availability regulates SIRT1 through a forkhead-dependent pathway. Science 2004, 306, 2105-2108.

82. Nisoli, E.; Tonello, C.; Cardile, A.; Cozzi, V.; Bracale, R.; Tedesco, L.; Falcone, S.; Valerio, A.; Cantoni, O.; Clementi, E.; et al. Calorie restriction promotes mitochondrial biogenesis by inducing the expression of eNOS. Science 2005, 310, 314-317. 
83. Feige, J.N.; Auwerx, J. Transcriptional targets of sirtuins in the coordination of mammalian physiology. Curr. Opin. Cell Biol. 2008, 20, 303-309.

84. Chen, Z.; Peng, I.C.; Cui, X.; Li, Y.S.; Chien, S.; Shyy, J.Y. Shear stress, SIRT1, and vascular homeostasis. Proc. Natl. Acad. Sci. USA 2010, 107, 10268-10273.

85. Mattagajasingh, I.; Kim, C.S.; Naqvi, A.; Yamamori, T.; Hoffman, T.A.; Jung, S.B.; DeRicco, J.; Kasuno, K.; Irani, K. SIRT1 promotes endothelium-dependent vascular relaxation by activating endothelial nitric oxide synthase. Proc. Natl. Acad. Sci. USA 2007, 104, 14855-14860.

86. Donato, A.J.; Magerko, K.A.; Lawson, B.R.; Durrant, J.R.; Lesniewski, L.A.; Seals, D.R. SIRT-1 and vascular endothelial dysfunction with ageing in mice and humans. J. Physiol. 2011, $589,4545-4554$.

87. Spallotta, F.; Cencioni, C.; Straino, S.; Nanni, S.; Rosati, J.; Artuso, S.; Manni, I.; Colussi, C.; Piaggio, G.; Martelli, F.; et al. A nitric oxide-dependent crosstalk between Class I and III histone deacetylases accelerates skin repair. J. Biol. Chem. 2013, 288, 11004-11012.

88. Migliaccio, E.; Giorgio, M.; Mele, S.; Pelicci, G.; Reboldi, P.; Pandolfi, P.P.; Lanfrancone, L.; Pelicci, P.G. The p66shc adaptor protein controls oxidative stress response and life span in mammals. Nature 1999, 402, 309-313.

89. Pelicci, G.; Lanfrancone, L.; Grignani, F.; McGlade, J.; Cavallo, F.; Forni, G.; Nicoletti, I.; Grignani, F.; Pawson, T.; Pelicci, P.G. A novel transforming protein (SHC) with an SH2 domain is implicated in mitogenic signal transduction. Cell 1992, 70, 93-104.

90. Ventura, A.; Luzi, L.; Pacini, S.; Baldari, C.T.; Pelicci, P.G. The p66Shc longevity gene is silenced through epigenetic modifications of an alternative promoter. J. Biol. Chem. 2002, 277, 22370-22376.

91. Chen, H.Z.; Wan, Y.Z.; Liu, D.P. Cross-talk between SIRT1 and p66Shc in vascular diseases. Trends Cardiovasc. Med. 2013, 14, doi:10.1016/j.tcm.2013.01.001.

92. Zaccagnini, G.; Martelli, F.; Fasanaro, P.; Magenta, A.; Gaetano, C.; di Carlo, A.; Biglioli, P.; Giorgio, M.; Martin-Padura, I.; Pelicci, P.G.; et al. p66ShcA modulates tissue response to hindlimb ischemia. Circulation 2004, 109, 2917-2923.

93. Zaccagnini, G.; Martelli, F.; Magenta, A.; Cencioni, C.; Fasanaro, P.; Nicoletti, C.; Biglioli, P.; Pelicci, P.G.; Capogrossi, M.C. p66(ShcA) and oxidative stress modulate myogenic differentiation and skeletal muscle regeneration after hind limb ischemia. J. Biol. Chem. 2007, 282, 31453-31459.

94. Di Stefano, V.; Cencioni, C.; Zaccagnini, G.; Magenta, A.; Capogrossi, M.C.; Martelli, F. p66ShcA modulates oxidative stress and survival of endothelial progenitor cells in response to high glucose. Cardiovasc. Res. 2009, 82, 421-429.

95. Giorgio, M.; Berry, A.; Berniakovich, I.; Poletaeva, I.; Trinei, M.; Stendardo, M.; Hagopian, K.; Ramsey, J.J.; Cortopassi, G.; Migliaccio, E.; et al. The p66Shc knocked out mice are short lived under natural condition. Aging Cell 2012, 11, 162-168.

96. Satoh, A.; Stein, L.; Imai, S. The role of mammalian sirtuins in the regulation of metabolism, aging, and longevity. Handb. Exp. Pharmacol. 2011, 206, 125-162.

97. Baur, J.A.; Sinclair, D.A. Therapeutic potential of resveratrol: The in vivo evidence. Nat. Rev. Drug Discov. 2006, 5, 493-506.

98. Chung, S.; Yao, H.; Caito, S.; Hwang, J.W.; Arunachalam, G.; Rahman, I. Regulation of SIRT1 in cellular functions: Role of polyphenols. Arch. Biochem. Biophys. 2010, 501, 79-90. 
99. Milne, J.C.; Lambert, P.D.; Schenk, S.; Carney, D.P.; Smith, J.J.; Gagne, D.J.; Jin, L.; Boss, O.; Perni, R.B.; Vu, C.B.; et al. Small molecule activators of SIRT1 as therapeutics for the treatment of type 2 diabetes. Nature 2007, 450, 712-716.

100. Motta, M.C.; Divecha, N.; Lemieux, M.; Kamel, C.; Chen, D.; Gu, W.; Bultsma, Y.; McBurney, M.; Guarente, L. Mammalian SIRT1 represses forkhead transcription factors. Cell 2004, 116, 551-563.

101. Minor, R.K.; Baur, J.A.; Gomes, A.P.; Ward, T.M.; Csiszar, A.; Mercken, E.M.; Abdelmohsen, K.; Shin, Y.K.; Canto, C.; Scheibye-Knudsen, M.; et al. SRT1720 improves survival and healthspan of obese mice. Sci. Rep. 2011, 1, doi:10.1038/srep00070.

102. Mai, A.; Valente, S.; Meade, S.; Carafa, V.; Tardugno, M.; Nebbioso, A.; Galmozzi, A.; Mitro, N.; de Fabiani, E.; Altucci, L.; et al. Study of 1,4-dihydropyridine structural scaffold: Discovery of novel sirtuin activators and inhibitors. J. Med. Chem. 2009, 52, 5496-5504.

103. Caton, P.W.; Nayuni, N.K.; Kieswich, J.; Khan, N.Q.; Yaqoob, M.M.; Corder, R. Metformin suppresses hepatic gluconeogenesis through induction of SIRT1 and GCN5. J. Endocrinol. 2010, 205, 97-106.

104. Li, Q.; Xiao, H.; Isobe, K. Histone acetyltransferase activities of cAMP-regulated enhancer-binding protein and p300 in tissues of fetal, young, and old mice. J. Gerontol. A Biol. Sci. Med. Sci. 2002, 57, B93-B98.

105. Bandyopadhyay, D.; Okan, N.A.; Bales, E.; Nascimento, L.; Cole, P.A.; Medrano, E.E. Down-regulation of p300/CBP histone acetyltransferase activates a senescence checkpoint in human melanocytes. Cancer Res. 2002, 62, 6231-6239.

106. Kang, H.L.; Benzer, S.; Min, K.T. Life extension in Drosophila by feeding a drug. Proc. Natl. Acad. Sci. USA 2002, 99, 838-843.

107. Tao, D.; Lu, J.; Sun, H.; Zhao, Y.M.; Yuan, Z.G.; Li, X.X.; Huang, B.Q. Trichostatin A extends the lifespan of Drosophila melanogaster by elevating hsp22 expression. Acta Biochim. Biophys. Sin. 2004, 36, 618-622.

108. Zhao, Y.; Sun, H.; Lu, J.; Li, X.; Chen, X.; Tao, D.; Huang, W.; Huang, B. Lifespan extension and elevated hsp gene expression in Drosophila caused by histone deacetylase inhibitors. Exp. Biol. 2005, 208, 697-705.

109. Bush, E.W.; McKinsey, T.A. Protein acetylation in the cardiorenal axis: The promise of histone deacetylase inhibitors. Circ. Res. 2010, 106, 272-284.

110. McKinsey, T.A. Targeting inflammation in heart failure with histone deacetylase inhibitors. Mol. Med. 2011, 17, 434-441.

111. Ferguson, B.S.; Harrison, B.C.; Jeong, M.Y.; Reid, B.G.; Wempe, M.F.; Wagner, F.F.; Holson, E.B.; McKinsey, T.A. Signal-dependent repression of DUSP5 by class I HDACs controls nuclear ERK activity and cardiomyocyte hypertrophy. Proc. Natl. Acad. Sci. USA 2013, 110, 9806-9811.

112. Yuan, Y.; Wang, Q.; Paulk, J.; Kubicek, S.; Kemp, M.M.; Adams, D.J.; Shamji, A.F.; Wagner, B.K.; Schreiber, S.L. A small-molecule probe of the histone methyltransferase G9a induces cellular senescence in pancreatic adenocarcinoma. ACS Chem. Biol. 2012, 7, 1152-1157.

(C) 2013 by the authors; licensee MDPI, Basel, Switzerland. This article is an open access article distributed under the terms and conditions of the Creative Commons Attribution license (http://creativecommons.org/licenses/by/3.0/). 